\title{
Wirkfaktoren psychosozialer Behandlungen bei Sucht und komorbiden psychischen Störungen
}

Effective Psychosocial Treatment for Patients with Substance Use Disorders and Comorbid Psychiatric Disorders

\author{
Franz Moggi
}

Universitätsklinik für Psychiatrie und Psychotherapie, Universität Bern, Bern, Schweiz

Korrespondenz:

Prof. Dr. phil. Franz Moggi, EMBA

Universitätsklinik für Psychiatrie und Psychotherapie

Universtität Bern

Bolligenstrasse 111

3000 Bern 60

Schweiz

E-Mail: moggi@puk.unibe.ch

Tel. +41319309111

Fax +41 319309414 


\section{Zusammenfassung}

Gegenstand und Ziel: Es werden die wichtigsten Wirkfaktoren erfolgreicher psychosozialer Behandlungen von Patienten mit einer Komorbidität von psychischen Störungen und Sucht (Doppeldiagnosen) herausgearbeitet. Material und Methoden: $\underline{\text { In den drei wissenschaftlichen }}$ Datenbanken PubMed, PsycINFO und Web of Science wurden neuere Metaanalysen und umfassende Übersichtsarbeiten zur Wirksamkeit psychosozialer Behandlungen bei Doppeldiagnosepatienten gesucht und aus deren Ergebnisse das vorliegende „narrative review“ erarbeitet. Dabei wird näher auf die Studienergebnisse zu schweren und zu leichten Formen von Komorbidität eingegangen. Ergebnisse: Integrative, gestufte Behandlungsprogramme, die störungsspezifische Interventionen kombinieren, sind Kontrollgruppen (z.B. Wartegruppen,

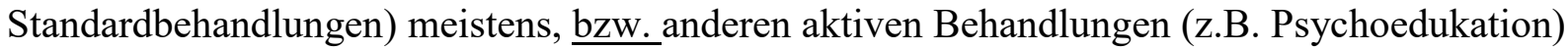
manchmal in den drei Ergebnisbereichen (Sucht, psychische Störung und Funktionsniveau) überlegen. Erfolgreiche Behandlungsprogramme enthalten meist motivierende Gesprächsführung, kognitiv-verhaltenstherapeutische Interventionen, suchtmittelreduzierende Interventionen wie Rückfallprävention oder Kontingenzmanagement und/oder Familieninterventionen. Schlussfolgerung: Die Heterogenität der Patienten-, Behandlungs-, Settings- und Ergebnismerkmale erschweren allgemeingültige Schlussfolgerungen über die Wirksamkeit psychosozialer Behandlungen. Bei Patienten mit Doppeldiagnosen sind integrierte Behandungsangebote am erfolgversprechendsten.

Schlüsselwörter: Komorbidität, Behandlungswirksamkeit, integrative Behandlungen, Übersicht 


\begin{abstract}
Objective: Results on the effectiveness of psychosocial treatments for patients with a comorbidity of psychiatric and substance use disorders (dual diagnosis patients) will be presented. Material and methods: PubMed, PsycINFO, and Web of science were searched for recent meta-analyses and comprehensive reviews on the efficacy of psychosocial treatments for dual diagnosis patients. The findings are presented in this narrative review for severe (e.g., schizophrenia) and for mild to moderate (e.g., anxiety disorders) dual diagnoses. Results: Promising treatments seem to be integrative stagewise programs that comprise motivational interviewing, cognitive-behavioral interventions, substance use reducing interventions such as relapse prevention and contingency management and/or family interventions. Such programs are mostly superior to control groups (e.g., waiting list) and they are sometimes superior to other active treatments (e.g., treatment as usual) in outcomes of substance use, psychiatric disorders and social functioning. Conclusions: Due to the heterogeneity in patients', treatments', settings', and outcomes' characteristics it is difficult to generally conclude which psychosocial treatments are effective. Integrated treatments seem to be most effective for dual diagnosis patients.
\end{abstract}

Key words: comorbidity, treatment effectiveness, integrated treatment, review 


\section{Wirkfaktoren psychosozialer Behandlungen bei Sucht und komorbiden psychischen Störungen}

Als vor über 25 Jahren systematisch mit der wissenschaftlichen Untersuchung der Wirksamkeit von Behandlungen von Patienten mit einer Komorbidität von psychischen Störungen (PS) und Substanzkonsumstörungen (SKS) begonnen wurde, bezeichneten einzelne Autoren die Therapie dieser sogenannten Doppeldiagnosepatienten (DDP) als eine „mission impossible“ (1). Tatsächlich zeigten fühere, meist unkontrollierte Studien, dass DDP in herkömmlichen Behandlungen im Vergleich zu Patienten ohne Komorbiditäten deutlich schlechtere Therapieverläufe (z.B. häufig und rasche Therapieabbrüche) und -ergebnisse (z.B. hohe Rückfall- und Rehospitalistionsraten), niedrigere Kennwerte psychosozialen Funktionierens wie hohe Raten an Arbeits- und Obdachlosigkeit sowie häufiger somatische Begleiterkankungen aufwiesen (2). Erschwerend für die Behandlung ist bis heute auch die traditionelle Trennung in Behandlungssysteme für Substanzkonsumstörungen einerseits und für psychische Störungen andererseits, die jeweils eigene therapeutische Standards entwickelten und bemüht sind, ausschliesslich Patienten ohne Komorbidität in ihre Behandlungsangebote aufzunehmen (3). Es ist deshalb dringend notwendig, Wirkfaktoren effektiver Behandlungsansätze für DDP zu identifizieren, um sie in der Therapie beider Behandlungssysteme einzusetzen. Obwohl heute mehr oder weniger gut kontrollierte klinische Studien zur Wirksamkeit psychosozialer Behandlungen von DDP vorliegen (3), so dass einige Metaanalysen sowie umfassende Übersichtsarbeiten erschienen sind, kommen Chow und Kollegen (4) zur Einschätzung, dass es sich bei der Behandlung von DDP immer noch um eine „mission impossible“ handle. In der vorliegenden Überblicksarbeit werden auf der Grundlage neuerer Arbeiten Schlussfolgerungen zur wirksamen Behandlung von Doppeldiagnosen gezogen. Dabei werden die Studienergebnisse zu schweren und leichten Komorbiditätsformen vorgestellt, wobei zunächst kurz auf die Probelmatik der Studienhterogenität eingegangen wird.

\section{Material und Methode}

In den drei wissenschaftlichen Datenbanken PubMed, PsycINFO und Web of Science wurden neuere Metaanalysen und umfassende Übersichtsarbeiten zur Wirksamkeit psychosozialer Behandlungen bei DDP gesucht. Dabei wurden folgende Suchkriterien verwendet: Metaanalysis OR review; treatment OR psychosocial treatment; comorbidity OR dual diagnosis; substance use 
disorder OR drug use disorder OR alcohol use disorder; und die jeweilige psychische Störung (schizophrenia OR schizoaffective OR psychotic disorder; bipolar disorder; depression OR depressive disorder; anxiety disorder; posttraumatic stress disorder; personality disorder).

\section{Heterogenität der Studien und ihre Folgen}

Das entmutigende Verdikt einer unmöglichen Aufgabe mag aus wissenschaftlicher Sicht weniger in den Studienergebnissen begründet sein als vielmehr in der Art und Weise wie diese Resultate zustande gekommen sind. Das grösste Problem lässt sich mit dem Begriff der Heterogenität umschreiben. So unterscheiden sich die Untersuchungen in den Patienten-, Behandlungs-, Settings- und Ergebnisvariablen derart stark, dass es kaum möglich ist, zwei vergleichbare Studien zur selben Fragestellung zu finden. Beispielsweise haben De Witte und Kollegen (5) in einem Übersichtsartikel auf der Grundlage von gut kontrollierten Studien versucht, die notwendigen Behandlungskomponenten einer erfolgreichen psychosozialen Behandlung von Patienten mit Schizophrenie und SKS im ambulanten Setting herauszuarbeiten. Erstens konnten nur gerade acht von 14 randomisiert-kontrollierten Studien zu diesem Thema gefunden werden, die ausschliesslich mit Patienten mit einer Schizophrenie und einer SKS durchgeführt wurden. Dabei identifizierten De Witte und Kollegen in den Untersuchungen zwei verschiedene Behandlungstypen als Experimentalgruppen, die gegen eine Kontrollgruppe (z.B. Wartegruppe, Standardbehandlung) getestet wurden:

a) Hinzufügen einer oder mehrerer zusätzlichen Interventionen zu einem bestehenden Standardbehandlungsprogramm und

d) Angebot eines integrativen Behandlungsprogrammes.

Unter integrativen Behandlungsprogrammen (IBP) werden Programme verstanden, die Interventionen zur Behandlung der PS und der SKS zur selben Zeit, im selben Setting und durch dieselben Behandlungspersonen anbieten. Wiederum unterschieden sich sowohl die verschiedenen IBP als auch die einzelnen Interventionen innerhalb des IBP stark. Sie können in einer oder einer Kombination von motivierender Gesprächsführung (,motivational interviewing“; $\mathrm{MI})$, kognitiv-verhaltenstherapeutischen Interventionen bzw. -therapien (KVT), Rückfallprävention (RP), Kontingenzmanagement (KM), Case Management (CM) oder Familieninterventionen (FI) bestehen. IBP gelten als die wirksamste Behandlung für DDP im 
Vergleich zur parallelen oder gar seriellen Behandlung der beiden Störungen, weil in ihnen die Interventionen für PS und SKS auf die individuellen Bedürfnisse der Patienten abgestimmt werden (3).

Ein weiteres Problem ist die Heterogenität der Patienten, insbesondere der Diagnosen, wenn von DDP gesprochen wird. Rosenthal und Westreich (6) versuchten eine Systematik in die verschiedenen Diagnosekombinationen von PS und SKS einzuführen, indem sie entlang des Schweregrades der Psychopathologie und der Sucht die Diagnosen einteilten. So definierten sie beispielsweise schwere DDP, wenn Patienten unter einer Psychose und unter Mehrfachabhängigkeit von Substanzen litten und leichte DDP, wenn die Patienten z.B. eine spezifische Spinnenphobie und Cannabismissbrauch aufwiesen. Allerdings hat diese Einteilung bis heute weder für Diagnostik, Ätiologie und Indikation noch für die Behandlung deutliche Vorteile gebracht, zumal der grösste Teil der Forschung zur Wirksamkeit psychosozialer Behandlungen mit Patienten mit schweren psychischen Störungen (v.a. Schizophrenie, schizoaffektive Störungen, bipolare Störungen, schwere Depressionen) durchgeführt wurden. In den Übersichtsartikeln wurde festgestellt, dass trotz der Grösse des Problems eine erstaunliche Armut in der Untersuchung von Patienten mit leichteren DDP vorliegt. Die Idee einer allgemein gültigen bzw. einheitlichen psychosozialen Behandlung für DDP wird letztlich fallengelassen (3).

\section{Komorbidität von schweren psychischen Störungen und Sucht}

\section{Schizophrenien und andere psychotische Störungen}

Unter schweren psychischen Störungen werden meistens psychotische Störungen, einschliesslich Schizophrenie, schizophrenieforme und schizoaffektive Störungen, bipolare Störungen und schwere Depressionen verstanden (3). Drake und Kollegen $(7,8)$ haben wiederholt systematische Übersichtsarbeiten zur Wirksamkeit von Behandlungsangeboten für schwere DDP, insbesondere schizophreniforme Störungen vorgestellt. Sie stellen dabei wiederholt fest, dass IBP, die ihren Schwerpunkt auf das Einbinden der Patienten durch MI (9) legen, erfolgreich sind, wenn sie auf der Grundlage des transtheoretischen Modells der Veränderungsphasen (TTM) ein gestuftes Programm zur Umsetzung und Stabilisierung von Verhaltensänderungen anbieten (10, 11). In ihrer umfassenden Übersicht über 45 kontrollierte Studien (22 experimentelle und 23 quasi-experimentelle Studien) identifizierten sie Gruppenangebote, KM und stationäre 
Langzeitprogramme als wirksame Interventionsformen zur Reduktion von Substanzkonsum und Verbesserungen in den psychischen Symptomen sowie des Funktionsniveaus (z.B. Verringerung der Hospitalisationsrate, Verbesserung der Therapieteilnahme, Wohn- und Arbeitsfähigkeit sowie Erhöhung der Lebensqualität). Zusätzlich zeigten Drake und Kollegen, dass CM im Vergleich zu anderen Interventionsformen (z.B. individuelle Beratung, intensive ambulante Rehabilitation) konsistent mit der Verbesserung des Funktionsniveaus korrelierte. Nur eine Studie untersuchte FI im Rahmen von MI und individualisierter KVT. FI zeigte in allen drei Ergebnisbereichen SKS, PS und Funktionsniveau deutliche Verbesserungen (12). Allerdings konnte jüngst in einer Studie mit grosser Fallzahl die Wirksamkeit von MI in Kombination mit KVT nicht belegt werden, so dass die Hypothese zu prüfen wäre, ob der Einbezug des sozialen Netzes (Familie, andere wichtigen Bezugspersonen) durch FI eine unerlässliche Behandlungskomponente erfolgreicher psychosozialer Behandlungen ist (13).

Cleary und Mitarbeiter (14) fanden ähnliche Resultate in ihrer systematischen Übersicht über 53 kontrollierte Studien (30 experimentelle und 23 quasi-experimentelle Studien). Wieder wies MI die beste Evidenz für die Reduktion des Substanzkonsums auf. Wenn KVT mit MI kombiniert wurde, ergaben sich auch Verbesserungen in den psychischen Symptomen. Stationäre Langzeitprogramme und KM zeigten konsistent einen positiven Zusammenhang mit der Reduktion des Substanzkonsums. Dass KVT nicht immer mit positiven Ergebnissen zusammenhängt, ist möglicherweise dadurch zu erklären, dass unter dem Begriff KVT eine Vielzahl von Interventionen zusammengefasst wird, die sich untereinander deutlich unterscheiden. Bei KVT handelt es sich um ein Psychotherapiemodell, nicht um eine spezifische Intervention.

De Witte und Mitarbeiter (5) schlossen in ihrer Übersicht zur ambulanten Behandlung von DDP mit Schizophrenie und SKS, dass umfassende, intensive und längere Programme, in denen Kombinationen mehrerer Interventionen (z.B. MI, KVT, RP, KT und FI) vorkommen, mit grösserer Wahrscheinlichkeit zu Verbesserungen in den drei Ergebnisbereichen SKS, PS und Funktionsniveau führen. Als erfolgreich haben sich die IBP „Behavioral Treatment for Substance Abuse in Severe and Persistent Mental Illness“ (BTSAS) (15) oder „Family Intervention for Dual Diagnosis“ (FIDD) (16) erwiesen. Beide Programme sind nach dem TTM aufgebaut und 
umfassen neben sozialen Fertigkeitentrainings und psychoedukativen Elementen auch MI, RP, KT bzw. Familieninterventionen.

Während die erwähnten Übersichtsarbeiten auch Studien enthalten, deren interne Validität wegen fehlender Randomisierung nicht immer gegeben ist, dafür aber über eine hohe externe Validität verfügen, zeigen Metaanalysen, die ausschliesslich randomisiert-kontrollierte Studien berücksichtigen, kaum Vorteile einzelner Interventionen oder IBP gegenüber ihren Kontrollgruppen $(4,17,18)$.

\section{Bipolare Störungen}

Wie bereits erwähnt, werden in den Studien zu schweren DDP oft auch Patienten mit Bipolaren Störungen als weitere Diagnosen zu Schizophrenie oder schizoaffektive Störungen mit einbezogen $(19,20)$. Bis heute gibt es nur schwache Belege, das IBP für Patienten mit bipolaren Erkrankungen und SKS wirksam sind. Allerdings sind auch nur zwei qualitativ akzeptable Studien zu dieser Störungskombination verfügbar (20,21). In einer Pilotstudie zeigten sich bei einem IBP im Vergleich zur Kontrollgruppe eine deutliche Verringerung in der Trinkmenge und den Symptomen der Manie, jedoch nicht der Depression (22). In einer zweiten Studie wurde die „Interpersonal and Social Rhyhtm Therapy“ (ISRT), die Patienten mit bipolaren Störungen darin fördert, den Zusammenhang zwischen Stimmungsveränderungen und zwischenmenschlichen Beziehungen zu verstehen, zirkadiane Rhythmen durch Tagesstrukturierung zu stabilisieren und psychische Symptome kontrollieren zu lernen (23). Verglichen mit einer Kontrollgruppe zeigte die ISRT positive Wirkung auf die Rückfallverhütung, die Verbesserung zwischenmenschlicher Beziehungen und die Lebensqualität im Vergleich zur Kontrollgruppe, die ausschliesslich Medikamente erhielt. Studien zu bipolarer Störung und SKS sind wie bei anderen Komorbiditätsformen jedoch deutlich unterrepräsentiert, so dass weitere Untersuchungen zur Absicherung notwendig sind.

\section{Komorbidität von leichten psychischen Störungen und Sucht}

\section{Depressionen}

Unter leichteren Formen von Komorbidität psychischer Störungen und Sucht werden Angststörungen und leichte bis mittelgradige Depressionen und Störungen durch 
Substanzkonsum verstanden (3). Bis heute sind nur gerade fünf kontrollierte Studien publiziert, welche die Behandlung von Depression und Sucht untersuchten, wobei alle hohe Ausfallraten bzw. kleine Patientenkollektive aufweisen (20, 24, 25). Die Untersuchungen ergaben, dass die Patienten der Experimentalgruppe im Vergleich zu denjenigen in der Kontrollgruppe eine stärkere Verbesserung im Substanzkonsum und in den Depressionssymptomen sowie eine grössere Veränderungsmotivation und einen längeren Verbleib in den Behandlungen aufwiesen. Allerdings ist auch für diese Komorbiditätsform die Heterogenität der Behandlungsansätze gross und reicht von einem IBP für Depression und SKS bis hin zu einer kognitivverhaltenstherapeutisch ausgerichteten Einzeltherapie, die zur Standardbehandlung hinzugefügt wurde. Des Weiteren unterscheiden sich inhaltlich nicht nur die Experimentalgruppen sondern auch die Kontrollgruppen deutlich (z.B. unterstützende Kurzzeitpsychotherapie).

Jüngst wurde in einer grossen randomisiert-kontrollierten Studie ein manualisiertes Behandlungsprogramm, in dem für eine Stichprobe von über 280 Patienten, die unter einer Depression und Alkoholmissbrauch litten, KVT kombiniert mit MI angeboten . Verglichen wurde die Wirksamkeit von Kurzintervention, Einzelpsychotherapie, die sich auf eine der beiden Komorbiditätsstörungen konzentrierte, oder integrative Einzelpsychotherapie, in deren Fokus beide Störungen waren (26). Die Patienten wurden zufällig zu einer der vier folgenden Bedingungen zugewiesen: eine 90 Minuten dauernde Kurzintervention, Kurzintervention gefolgt von neun einstündigen einzelpsychotherapeutischen Sitzungen, die sich entweder auf den Alkoholmissbrauch oder auf die Depression konzentrierten, oder Kurzintervention gefolgt von neun einstündigen integrativen Einzelpsychotherapiesitzungen zur Behandlung beider psychiatrischer Störungen. Verglichen mit der Kurzintervention wiesen alle drei Einzelpsychotherapien bessere Ergebnisse im Alkoholkonsum der Patienten auf. Verglichen mit den Einzelpsychotherapien, in denen nur eine Störung behandelt wurde, war die integrative Einzelpsychotherapie in Bezug auf den Alkoholkonsum und die Depressionssymptome erfolgreicher. Interessanterweise ergab sich ein geschlechtstypischer Unterschied. Innerhalb der Einzelpsychotherapien mit nur einem Störungsfokus war bei Männern die alkoholspezifische Therapie erfolgreicher als die depressionsspezifische, bei Frauen die depressionsspezifische Einzeltherapie erfolgreicher als die alkoholspezifische. Diese Studie zeigt nicht nur, dass das IPB zu besseren Ergebnisen als störungsspezifische Interventionen führt, sondern dass es innerhalb 
der störungsspezifischen Psychotherapie bei dieser Komorbiditätsform geschlechtstypische Unterschiede gibt.

Diese gruppenspezifischen Kurzzeitergebnisse waren jedoch in der Form nicht nachaltig. Baker und Kollegen (27) berichten andere Langzeitffekte zum Zeitpunkt der 6-, 12-, 24- und 36Monatskatamnese. Verglichen mit der Kurzintervention waren alle drei Einzelpsychotherapien in der Katmnese erfolgreicher in Bezug auf die Reduktion von Depressionssymptomen und die Verbesserung des Funktionsniveaus. Die alkohol- und die depressionsfokussierten Therapien waren in Bezug auf die Reduktion von Depressionssymptomen gleich wirksam, wobei die alkoholfokussierte Therapie wirksamer den Alkoholkonsum verringerte. Baker und Kollegen schlossen aus ihren Ergebnissen, dass zwar initial beide Störung zu behandeln seien, dass aber im weiteren Verlauf zusätzlich integrative oder alkoholfokussierte Therapien hinzukommen müssen, um langfristig die Komorbidität von Depression und Sucht erfolgreich zu behandeln.

In einer Metaanalyse über 12 kontrollierte Studien mit insgesamt 1'721 Patienten mit Depressionen und Alkoholkonsumstörungen zur Frage, ob die Kombination von Standardbehandlung, KVT und MI im Vergleich zur Standardbehandlung alleine bessere Ergebnisse aufweisen, konnten Riper und Kollegen (28) zeigen, dass die Kombinationsbehandlung der Standardbehandlung mit kleinen Effektstärken von $g=0.17$ in der Reduktion von Alkoholkonsum und von $\mathrm{g}=0.27$ in der Reduktion von Depressionssymptomen leicht überlegen ist. Internet gestützte Inteventionen waren bei der Verbesserung der Depressionssymptome gar face-to-face Interventionen signifikant überlegen ( $\mathrm{g}=0.73$ vs. $\mathrm{g}=$ 0.23 ), wobei nur zwei der eingeschlossenen Studien über Internet gestütze Interventionen verfügten.

\section{Angststörungen}

In einer Übersicht zur Behandlung von Angststörungen und Sucht fand Hesse (24), dass IBP im Allgemeinen die Abstinenztage erhöht, tendenziell die Angstsymptome verringert und die Haltequote verbessert. Er schloss daraus, dass psychosoziale Interventionen alleine für eine erfolgreiche Behandlung bei dieser Komorbiditätsform jedoch nicht genügen und dass dafür andere Interventionen als die bisher vorgestellten benötigt werden. Ähnliche Schlussfolgerungen wurden in einer anderen umfassenden Übersichtsarbeit gezogen (21). Allerdings unterschieden 
sich die Studien nicht nur in den Behandlungsprogrammen sondern auch in den Angstdiagnosen (z.B. Panikstörung, soziale Ängstlichkeit, Zwangsstörungen oder posttraumatische Belastungsstörungen) und den Suchterkrankungen (z.B. nur Alkoholabhängigkeit bzw. Drogenabhängigkeit) deutlich. Ebenfalls gab es Unterschiede in dem Behandlungssettings, die von stationärer Therapie über therapeutische Gemeinschaften bis hin zu ambulanten Therapien reichten, so dass eine allgemeine Schlussfolgerung über die Wirksamkeit von Behandlungsprogrammen bei Komorbidität von Angst und Sucht schwer zu rechtfertigen wäre.

Jüngst haben Kushner und Kollegen (29) in einer randomisiert-kontrollierten Studie an 344 Patienten mit einer Alkoholkonsumstörung und einer komorbiden Sozialen Phobie, Panikstörung oder Generalisierten Angststörung, zeigen können, dass die Patienten in einer dreiwöchigen Standardalkoholbehandlung, die zusätzlich eine integrative, kognitiv-verhaltenstherapeutische Interventionsgruppe zur Angsstörung und zum Zusammenhang zwischen Angst- und Alkoholkonsumstörung besuchten, bessere Ergebnisse in Bezug auf die Reduktion des Alkoholkonsums aufwiesen als Patienten in der Vergleichsgruppe, die ein Entspannungstraining anstelle des integrativen Interventionsgruppe absolvierten. Die Angststörung verbesserte sich in beiden Gruppen, aber die Patienten in der integrierten Interventionsgruppe wiesen vier Monate nach Behandlungsende signifikant bessere Angstwerte auf als die Patienten der Entspannungsgruppe. Möglicherweise hilft die integrative Interventionsgruppe die Ergebnisse der Standardalkoholbehandlung zu verbessern, die u.a. wegen der Reduktion des Alkoholkonsums eine Wirkung auf die Verbesserung der Angststörung zeigt.

\section{Posttraumatische Belastungsstörung}

In der Komorbiditätsforschung nimmt die posttraumatische Belastungsstörung (PTBS) eine Sonderstellung ein, da sie die am häufigsten untersuchte Angststörung ist. Ihre Behandlung wird in die drei Phasen Stabilisierung, Konfrontation und Reintegration eingeteilt, wobei in der Konfrontationsphase die Exposition als die Standardintervention angesehen wird. Obwohl Kliniker empfehlen, die traumafokussierte Exposition erst nach erfolgreicher psychischer Stabilisierung anzuwenden, werden in der Therapieforschung dieser Komorbiditätsform zunehmen IBP untersucht, die bereits früh in der Behandlung gleichzeitig mit Interventionen zur Reduktion des Suchtmittelkonsums mit der Exposition beginnen. Schäfer und Kollegen unterscheiden in ihrer Übersicht IBP für die Stabilisierungs- und IBP für die 
Konfrontationsphase (30); in Übersichtsarbeiten und Metaanalysen aus dem englischsprachigen Raum wird zwischen nicht-traumafokussierten und traumafokussierten Therapien unterschieden (31-33). Bis heute scheint es keine Studien zur Reintegrationsphase zu geben.

Für die Stabilisierungsphase gibt es ein häufig untersuchtes, mittlerweile etabliertes und auch im deutschen Sprachraum bekanntes Behandlungsprogramm: das Therapiemanual „Sicherheit finden“" wurde von Najavits (34) vorgestellt, das in einigen randomisiert-kontrollierten Untersuchungen einschliesslich Multizenterstudien im Vergleich zu Kontrollgruppen bessere Ergebnisse in den PTBS Symptomen und der SKS zeigte. Allerdings war das Programm im Langzeitverlauf einem suchtmittelbezogenen Rückfallpräventionsprogramm nicht überlegen (21, $35)$.

Für die Konfrontationsphase wurden mehrere IBP vorgestellt, die meist in unkontrollierten und an Fallzahlen kleinen Studien $(\mathrm{N}<47)$ evaluiert wurden $(36,37)$. Eine Ausnahmen ist z.B. eine randomisiert-kontrollierte Studie von Sannibale und Kollegen (38) mit insgesamt 62 Patienten und zwei verschiedenen kognitiv-verhaltenstherapeutische Interventionsgruppen, die innerhalb von 12 Wochen entweder wöchentlich zusätzlich eine integrative Einzelsitzung zu PTBS und Alkoholkonsumstörungen einschliesslich Traumakonfrontation oder lediglich zusätzlich eine supportive Beratung erhielten. Beide Gruppen verbesserten signifikant sich in den Ergebnisvariablen Alkoholkonsum, Angst- und Depressionssymptome sowie im Schweregrad der PTBS Symptomatik, wobei die Verbesserung der integrativen Gruppe mit Traumaexpostion in Bezug auf die PTBS Symptomatik in den 5- und 9-Monatskatamnesen doppelt so stark ausfiel wie in der supportiven Beratungsgruppe, die jedoch bessere Ergebnisse in der Verringerung ihres Alkoholkonsums zeigte. Ähnliche Ergebnisse fanden früher Mills und Kollegen (39), in ihrer randomisiert-kontrollierten Studie mit 103 Patienten, wobei sich die beiden Gruppen (IBP mit Traumakonfrontation vs. Standardbehandlung) in der Verbesserung des Alkoholkonsums nicht unterschieden. Allerdings wurden in den Katamnesen nach sechs Wochen bzw. drei und neun Monaten nach der Behandlung keine Gruppenunterschiede gefunden.

Jüngst haben Roberts und Kollegen $(31,32)$ die Ergebnisse ihrer Cochrane Metaanalyse zu den verfügbaren randomisiert-kontrollierten Studien zur Behandlung der Komorbidität von PTBS und SKS vorgestellt. Sie konnten 13 Studien mit 1'506 Patienten in ihre quantitative Analyse 
einschliessen, wobei sie zwischen nicht-traumafokussierten und traumafokussierten Studien im Vergleich zu Standardtherapie, Minimaltherapie und andere aktive Therapie unterschieden. Einzeltherapien mit trauma- und substanzfokussierten Therapiekomponenten waren wirksamer als Standard- und Minimaltherapien. In Bezug auf die PTBS Symptomatik zeigten sie positivere Kurzzeit- und Langzeiteffekte und in Bezug auf den Substanzkonsum nur positivere Langzeiteffekte. Einzeltherapie mit traumafokussierte Komponenten zeigten keine besseren Effekte auf PTBS Symptomatik als lediglich substanzfokussierte Therapien. Nichttraumafokussierte Therapien, ob Einzel- oder Gruppentherapien, waren nicht wirksamer als substanzfokussierte Therapien oder Standard- und Minimaltherapien. Die Patientenzahlen und die Effektstärken waren klein, der Katamnesezeitraum kurz und die Ausfallrate der Patienten und die Heterogenität der Studien hoch, so dass die Ergebnisse nicht generalisiert werden können (31, $32)$.

\section{Persönlichkeitsstörungen und Sucht}

Für Persönlichkeitsstörungen ist die psychosoziale Behandlung, insbesondere störungsspezifische Psychotherapie, die Behandlung der ersten Wahl. In Bezug auf die Komorbidität von Persönlichkeitsstörungen und Sucht liegen ebenfalls sehr wenig randomisiert-kontrollierte Studien vor. Der Schwerpunkt liegt auf der Untersuchung der Borderline Persönlichkeitsstörung. In systematischen Übersichtsarbeiten identifizierten Euler und Kollegen, Lee und Kollegen und Pennay und Kollegen drei verschiedene Psychotherapiemodelle, die in wenigen wissenschaftlich akzeptablen Studien (Bandbreite zwischen 6 und 10 Studien) untersucht wurden (40-42): Die „Dialectic Behavioral Therapy“ (DBT) (43), die „Dynamic Deconstructive Psychotherapy“ (DyDP) (44) und die „Dual Focus Schema Therapie“ (DFST) (45). Die Ergebnisse zu DBT zeigen im Allgemeinen Verbesserungen im Substanzkonsum, in den Symptomen der Persönlichkeitsstörung und im Funktionsniveau. Während die DBT der Standardbehandlung meist überlegen war, schnitten sie jedoch $m$ Vergleich zu achtsamkeitsbasierten Intervnetionen in Kombination mit Zwölf-Schritte-Programme nicht besser ab. Die DyDP wies im Vergleich zur Kontrollgruppe bessere Ergebnisse für das Suchtverhalten und die Symptome der Persönlichkeitsstörung auf. Schliesslich zeigte die DFST bei verschiedenen

Persönlichkeitsstörungen zwar vielversprechende Ergebnisse in Bezug auf den Substanzkonsum, aber diese Ergebnisse beruhen hohen Ausfallraten. Hinzu kommt, dass in den meisten Untersuchungen kleine Fallzahlen aufweisen und die Patienten nicht nur in Bezug auf die 
Persönlichkeitsstörung Symptome, sondern auch unter anderen komorbiden psychische Störungen wie z.B. Depression oder Angststörungen leiden. Qualitativ sind die DBT Studien etwas besser als die DyDP und v.a. die DFST Studien. Auch im Persönlichkeitsstörungsbereich sind dringend weitere gute Studien erforderlich, um gesicherte Empfehlungen für eine wirksame Therapie abgeben zu können, wobei zum heutigen Zeitupunkt die DBT am meisten positive Evidenz aufweist.

\section{Schlussfolgerungen und Perspektiven}

In diesem Kapitel zur Komorbidität psychischer Störungen und Sucht wurde versucht, sich ein Bild darüber zu verschaffen, welche psychosozialen Therapien und pharmakologischen Behandlungen für DDP wirksam sind, indem Metaanalysen und umfassende Übersichtsarbeiten über kontrollierte klinische Studien berücksichtigt wurden. Die Studien sind schwer miteinander vergleichbar, weil sie äusserst heterogen in den Patienten-, Programm- und Interventions-, Setting-, Intensitäts- und Ergebnismerkmalen sind. Ebenfalls wurden die ganz wenigen kontrollierten Interventionsstudien zu Kombinationsstherapien mit psychosozialen und pharmakologischen Behandlungen nicht berücksichtigt. Aus wissenschaftlicher Perspektive macht das gesicherte Schlussfolgerung sehr schwierig. Hinzu kommt, dass die Studien vorwiegend aus den USA, einige aus Canada, Australien oder Grossbritannien, aber nur wenige aus Kontinentaleuropa stammen $(46,47)$. Im deutschen Sprachraum sind nur einige Behandlungsmanuale $(34,48-51)$ zu oder umfassende Monographien $(2,52)$ zu DDP erschienen.

Wollten wir versuchen, eine vorsichtige Schlussfolgerung zu formulieren, so kann man festhalten, dass IBP mit mehreren störungsspezifischen Interventionen eher wirksam sind und im besten Fall in allen Ergebnisvariablen den Kontrollgruppen bzw. den Standardbehandlungen überlegen sind. Ein breiteres IBP ist wahrscheinlich effektiver als das blosse Hinzufügen einer einzelnen Intervention (z.B. Rückfallprävention ) zu einer Standardbehandlung. Wenn die Patienten unter einer schweren DDP mit niedrigem Funktionsniveau leiden, ist eine stationäre Behandlung notwendig, selten genügt eine intensive ambulante Therapie. Nicht nur integrative auch parallele, aber nicht sequenzielle Behandlungen können insbesondere dann wirksam sein, wenn die Interventionen erfolgreich in der Reduktion oder Stabilisierung des Substanzkonsums sind. Stufenprogramme, die sich an die Veränderungsphasen des TTM anlehnen, sind zwar 
aufwändig aber eher erfolgreich. Mehrere Behandlungskomponenten von IBP wie z.B. MI, KVT, RP, KM und FI haben sich wiederholt als effektiv erwiesen. So gesehen ist die Behandlung von DDP ist keine unmögliche wie zu Beginn dieses Kapitels erwähnt, aber eine auwändige und schwierige Aufgabe. Zukünftige Studien sollten sich darauf konzentrieren, gestufte IBP zu untersuchen, die MI in Kombination mit KVT und Interventionen zur Reduktion des Substanzkonsums (z.B. RP, KT) enthalten, den Einbezug des sozialen Umfeldes der Patienten berücksichtigen sowie für DDP wirksame Medikamente kombinieren (3).

\section{Fazit für die Praxis}

Integrative, nach Intensität gestufte Behandlungsprogramme, die störungsspezifische Interventionen geschickt kombinieren und motivierende Gesprächsführung, kognitivverhaltenstherapeutische Interventionen, suchtmittelreduzierende Interventionen wie Rückfallprävention oder Kontingenzmanagement und/oder Familieninterventionen enthalten, sowie die richtige Medikation verwenden, scheinen weniger aufwändigen Behandlungen in Bezug auf die Verbesserung im Substanzkonsum, in Symptomen der psychischen Störungen und im Funktionsniveau überlegen zu sein (3). 


\section{Abkürzungsverzeichnis}

BTSAS Behavioral Treatment for Substance Abuse in Severe and Persistent Mental Illness

CM Case Management

DBT Dialectic Behavioral Therapy

DDP Doppeldiagnosepatienten

DFST Dual Focus Schema Therapie

DyDP Dynamic Deconstructive Psychotherapy

FI Familienintervention(en)

FIDD Family Intervention for Dual Diagnosis

IBP Integrative(s) Behandlungsprogramm(e)

ISRT Interpersonal and Social Rhyhtm Therapy

KM Kontingenzmanagement

KVT Kognitiv-verhaltenstherapeutische Intervention(en) bzw. -therapi(en)

MI Motivierender Gesprächsführung (,motivational interviewing“)

PS Psychische Störung(en)

PTBS Posttraumatische Belastungsstörung

RP Rückfallprävention

SKS Substanzkonsumstörungen

TTM Transtheoretisches Modell 


\section{Literatur}

1. Roberts LJ, Shaner A, Eckman TA, Tucker DE, Vaccaro JV. Effectively treatment of stimulant-abusing schizophrenics: Mission impossible? New Directions for Mental Health Services. 1992;53:55-65.

2. Moggi F, (Hrsg). Doppeldiagnosen. Komorbidität psychischer Störungen und Sucht. 2 ed. Bern: Huber; 2007.

3. Moggi F, Öjehagen A. Evidence-supported Psychosocial Treatment for Dual Disorder Patients. In: Dom G, Moggi F, (Hrsg). Co-occurring Addictive and Psychiatric Disorders. 1st. ed. Heidelberg: Springer; 2015. p. 261-77.

4. Chow CM, Wieman D, Cichocki B, Qvicklund H, Hiersteiner D. Mission impossible: treating serious mental illness and substance use co-occurring disorder with integrated treatment: a meta-analysis. Mental Health and Substance Use. 2012:1-19.

5. De Witte NAJ, Crunelle CL, Sabbe B, Moggi F, Dom G. Treatment for outpatients with comorbid schizophrenia and substance use disorders: a review. European Addiction Research. 2014;20:105-14.

6. Rosenthal RN, Westreich L. Treatment of persons with dual diagnoses of substance use disorder and other psychological problems. In: McCrady BS, Epstein EE, (Hrsg). Addictions A comprehensive guidebook. New York: Oxford University Press; 1999. p. 439-76.

7. Drake RE, Mueser KT, Brunette MF, McHugo GJ. A review of treatments for people with severe mental illnesses and co-occurring substance use disorders. Psychiatric Rehabilitation Journal. 2004;27(4):360-74. Epub 2004/06/30.

8. Drake RE, O'Neal EL, Wallach MA. A systematic review of psychosocial research on psychosocial interventions for people with co-occurring severe mental and substance use disorders. Journal of Substance Abuse Treatment. 2008;34(1):123-38. Epub 2007/06/19.

9. Miller WR, Rollnick S. Motivierende Gesprächsführung. 3. Aufl. Freiburg: Lambertus; 2009.

10. Osher FC, Kofoed LL. Treatment of patients with psychiatric and psychoactive substance use disorders. Hospital and Community Psychiatry. 1989;40:1025-35.

11. Prochaska JO, DiClemente CC, Norcross JC. In search how people change. Applications to addictive behaviors. American Psychologist. 1992;47:1102-14. 
12. Haddock G, Barrowclough C, Tarrier N, Moring J, O'Brien R, Schofield N, et al. Cognitivebehavioral therapy and motivational intervention for schizophrenia and substance misuse. British Journal of Psychiatry. 2003;183:418-26.

13. Barrowclough C, Haddock G, Wykes T, Beardmore R, Conrod P, Craig T, et al. Integrated motivational interviewing and cognitive behavioural therapy for people with psychosis and comorbid substance misuse: randomised controlled trial. BMJ. 2010;341:c6325. Epub 2010/11/26.

14. Cleary M, Hunt GE, Matheson S, Walter G. Psychosocial treatments for people with cooccurring severe mental illness and substance misuse: systematic review. Journal of Advanced Nursing. 2009;65(2):238-58. Epub 2008/11/20.

15. Bellack AS, Bennett ME, Gearon JS, Brown CH, Yang Y. A randomized clinical tral of a new behavioral treatment for drug abuse in people with severe and persistent mental illness. Archives of General Psychiatry. 2006;63:426-32.

16. Mueser KT, Glynn SM, Cather C, Xie H, Zarate R, Smith LF, et al. A randomized controlled trail of Family Intervention for Co-occurring Substance use and Severe Psychiatric Disorders. Schizophrenia Bulletin. 2013;39(658-672).

17. Cleary M, Hunt GE, Matheson SL, Siegfried N, Walter G. Psychosocial interventions for people with both severe mental illness and substance misuse (Review). The Cochrane Library. 2010; (3).

18. Jeffrey DP, Ley A, McLaren S, Siegfried N. Psychosocial treatment programmes for people with both severe mental illness and substance misuse (Review). The Cochrane Library. 2007; (4).

19. Horsfall J, Cleary M, Hunt GE, Walter G. Psychosocial treatments for people with cooccurring severe mental illnesses and substance use disorders (dual diagnosis): a review of empirical evidence. Harvard Review of Psychiatry. 2009;17(1):24-34. Epub 2009/02/12.

20. Tiet QQ, Mausbach B. Treatments for patients with dual diagnosis: a review. Alcoholism: Clinical and Experimental Research. 2007;31(4):513-36. Epub 2007/03/22.

21. Kelly TM, Daley DC, Douaihy AB. Treatment of substance abusing patients with comorbid psychiatric disorders. Addict Behav. 2012;37(1):11-24. Epub 2011/10/11.

22. Frank E, Swartz HA, Kupfer DJ. Interpersonal and social rhythm therapy: managing the chaos of bipolar disorder. Biological psychiatry. 2000;48(6):593-604. Epub 2000/10/06. 
23. Weiss RD, Griffin ML, Greenfield SF, Najavits LM, Wyner D, Soto JA, et al. Group therapy for patients with bipolar disorder and substance dependence: results of a pilot study. The Journal of Clinical Psychiatry. 2000;61(5):361-7. Epub 2000/06/10.

24. Hesse M. Integrated psychological treatment for substance use and co-morbid anxiety or depression vs. treatment for substance use alone. A systematic review of the published literature. BMC Psychiatry. 2009;9:6. Epub 2009/02/24.

25. Murthy P, Chand P. Treatment of dual diagnosis disorders. Current Opinion in Psychiatry. 2012;25(3):194-200. Epub 2012/03/08.

26. Baker AL, Kavanagh DJ, Kay-Lambkin FJ, Hunt SA, Lewin TJ, Carr VJ, et al. Randomized controlled trial of cognitive-behavioural therapy for coexisting depression and alcohol problems: short-term outcome. Addiction. 2010;105(1):87-99. Epub 2009/11/19.

27. Baker AL, Kavanagh DJ, Kay-Lambkin FJ, Hunt SA, Lewin TJ, Carr VJ, et al. Randomized controlled trial of MICBT for co-existing alcohol misuse and depression: outcomes to 36months. J Subst Abuse Treat. 2014;46(3):281-90. Epub 2013/11/12.

28. Riper H, Andersson G, Hunter SB, de Wit J, Berking M, Cuijpers P. Treatment of comorbid alcohol use disorders and depression with cognitive-behavioural therapy and motivational interviewing: a meta-analysis. Addiction. 2014;109(3):394-406. Epub 2013/12/07.

29. Kushner MG, Maurer EW, Thuras P, Donahue C, Frye B, Menary KR, et al. Hybrid cognitive behavioral therapy versus relaxation training for co-occurring anxiety and alcohol disorder: a randomized clinical trial. Journal of Consulting and Clinical Psychology. 2013;81(3):429-42. Epub 2013/01/02.

30. Schäfer I, Schulze C, Stubenvoll M. Psychotherapie bei Abhängigkeitserkrankungen und Posttraumatischer Belastungsstörung. Sucht. 2011;57(5):353-61.

31. Roberts NP, Roberts PA, Jones N, Bisson JI. Psychological interventions for post-traumatic stress disorder and comorbid substance use disorder: A systematic review and meta-analysis. Clinical Psychology Review. 2015;38:25-38. Epub 2015/03/21.

32. Roberts NP, Roberts PA, Jones N, Bisson JI. Psychological therapies for post-traumatic stress disorder and comorbid substance use disorder. The Cochrane database of systematic reviews. 2016;4:CD010204. Epub 2016/04/05.

33. van Dam D, Ehring T, Vedel E, Emmelkamp PM. Trauma-focused treatment for posttraumatic stress disorder combined with CBT for severe substance use disorder: a randomized controlled trial. BMC Psychiatry. 2013;13:172. Epub 2013/06/21. 
34. Najavits LM, Schäfer I, Stubenvoll M, Dilling A. Posttraumatische Belastungsstörung und Substanzmissbrauch: Das Therapieprogramm «Sicherheit finden». Göttingen: HogrefeVerlag; 2008.

35. Hien DA, Cohen LR, Miele GM, Litt LC, Capstick C. Promising treatments for women with comorbid PTSD and substance use disorders. The American Journal of Psychiatry. 2004;161(8):1426-32. Epub 2004/08/03.

36. Torchalla I, Nosen L, Rostam H, Allen P. Integrated treatment programs for individuals with concurrent substance use disorders and trauma experiences: a systematic review and metaanalysis. J Subst Abuse Treat. 2012;42(1):65-77. Epub 2011/11/01.

37. van Dam D, Vedel E, Ehring T, Emmelkamp PM. Psychological treatments for concurrent posttraumatic stress disorder and substance use disorder: a systematic review. Clinical Psychology Review. 2012;32(3):202-14. Epub 2012/03/13.

38. Sannibale C, Teesson M, Creamer M, Sitharthan T, Bryant RA, Sutherland K, et al. Randomized controlled trial of cognitive behaviour therapy for comorbid post-traumatic stress disorder and alcohol use disorders. Addiction. 2013;108(8):1397-410.

39. Mills KL, Teesson M, Back SE, Brady KT, Baker AL, Hopwood S, et al. Integrated exposure-based therapy for co-occurring posttraumatic stress disorder and substance dependence: a randomized controlled trial. JAMA. 2012;308(7):690-9. Epub 2012/08/16.

40. Euler S, Sollberger D, Bader K, Lang UE, Walter M. A Persönlichkeitsstorungen und Sucht: Systematische Literaturubersicht zu Epidemiologie, Verlauf und Behandlung. 2015;83(10):544-54. Epub 2015/11/21.

41. Lee NK, Cameron J, Jenner L. A systematic review of interventions for co-occurring substance use and borderline personality disorders. Drug and Alcohol Review. 2015;34(6):663-72. Epub 2015/04/29.

42. Pennay A, Cameron J, Reichert T, Strickland H, Lee NK, Hall K, et al. A systematic review of interventions for co-occurring substance use disorder and borderline personality disorder. Journal of Substance Abuse Treatment. 2011;41(4):363-73.

43. Dimeff LA, Linehan MM. Dialectical behavior therapy for substance abusers. Addiction: Science \& Clinical Practice. 2008;4(2):39-47. Epub 2008/05/24.

44. Gregory RJ, Remen AL. A manual-based psychodynamic therapy for treatment-resistant borderline personality disorder. Psychotherapy (Chic). 2008;45(1):15-27. Epub 2008/03/01. 
45. Ball SA. Manualized treatment for substance abusers with personality disorders: dual focus schema therapy. Addictive behaviors. 1998;23(6):883-91. Epub 1998/11/05.

46. Moggi F, Brodbeck J, Költzsch K, Bachmann KM. One-year follow-up of dual diagnosis patients attending a 4-months integrative inpatient treatment. European Addiction Research. 2002;8:30-7.

47. Morrens M, Dewilde B, Sabbe B, Dom G, De Cuyper R, Moggi F. Treatment outcomes of an integrated residential programme for patients with schizophrenia and substance use disorder. European Addiction Research. 2011;17(3):154-63. Epub 2011/03/31.

48. D`Amelio R, Behrendt B, Wobrock T. Psychoedukation Schizophrenie und Sucht: Manual zur Leitung von Patienten- und Angehörigengruppen. 2 ed. Frankfurt: Urban \& Fischer; 2007.

49. Gouzoulis-Mayfrank E. Komorbidität Psychose und Sucht. Von den Grundlagen zur Praxis. Darmstadt: Steinkopf; 2007.

50. Moggi F, Donati R. Psychische Störungen und Sucht: Doppeldiagnosen. Schulte D, Grawe K, Hahlweg K, Vaitl D, (Hrsg). Göttingen: Hogrefe; 2004.

51. Walter M, Sollberger D, Euler S. Persönlichkeitsstörungen und Sucht. Risiken - FormenInterventionen. Stuttgart: Kohlhammer; 2015.

52. Walter M, Gouzoulis-Mayfrank E, (Hrsg). Psychische Störungen und Suchterkrankungen. Diagnostik und Behandlung von Doppeldiagnosen. Stuttgart: Kohlhammer; 2014. 